\title{
Some new types of associated curves in Euclidean 3-space
}

\author{
Sibel Özdoğan, O. Oğulcan Tuncer, İsmail Gök and Yusuf Yaylı
}

\begin{abstract}
In this paper, we introduce the concept of $D$-direction curve and $C$ direction curve of a given curve using the alternative frame $\{N, C, W\}$ in Euclidean 3-space. Furthermore, we define a $(N, C)$ plane curve, a $(N, W)$ plane curve and a $(C, W)$ plane curve of a given curve, and give some results related to them. Finally, we present some illustrated examples for new associated curves.
\end{abstract}

\section{Introduction}

The space curves in 3-dimensional Euclidean $\mathbb{E}^{3}$ is one of the most interesting and fundamental subjects in differential geometry. Hence, many authors defined some special curves and obtained characterizations of them. Among them, especially a general helix and slant helix are used for many applications. A general helix in Euclidean 3-space is defined that its tangent vector field makes a constant angle with a fixed direction called the axis of general helix. M.A. Lancret [7] in 1802 gave a useful result for general helices and it was first proved by B. de Saint Verant: A curve is a general helix if and only if

$$
H(s)=\frac{\tau(s)}{\kappa(s)}
$$

is a constant function, where $H(s)$ defined by [15] is called harmonic curvature of the curve. Then, Izumiya and Takeuchi [4] defined a new kind of helices

Key Words: Integral curve, general helix, slant helix, C-slant helix, associated curves. 2010 Mathematics Subject Classification: 53A04, 53A05.

Received: 08.04.2017

Revised: 08.07.2017

Accepted: 11.07.2017 
called slant helices in $\mathbb{E}^{3}$. A slant helix is defined with the property which the principal normal vector field of the curve makes a constant angle with a fixed line. Moreover, they gave an important result for slant helices. A curve is a slant helix iff the following relation is satisfied:

$$
\sigma=\frac{\kappa^{2}}{\left(\kappa^{2}+\tau^{2}\right)^{3 / 2}}\left(\frac{\tau}{\kappa}\right)^{\prime}=\text { const. }
$$

Moreover, Kula and Yaylı examined slant helices and their spherical indicatrices in [6]. Then, Okuyucu et al. [9] defined slant helices in three dimensional Lie groups with a bi-invariant metric. In [13], Uzunoglu et al. gave the slant helix characterization in terms of the harmonic curvature as

$$
\sigma=\frac{g}{f}=\frac{H^{\prime}}{\kappa\left(1+H^{2}\right)^{3 / 2}}=\text { const. }
$$

In the study of space curves, associated curves of a curve are very interesing curves. Recently, many authors have defined various associated curves of a curve such as Bertrand curves, Mannheim curves, pedal curves, offset curves. In [1], Choi and Kim defined principal (binormal)-direction curves and principal (binormal)-donor curves of Frenet curves in $\mathbb{E}^{3}$. Moreover, they introduced PD-rectifying curves and gave a new characterization of Bertrand curves via the PD-rectifying curves. Macit and Duldul defined $W$-direction curves and $W$-rectifying curves of a Frenet curve in $\mathbb{E}^{3}$ utilizing the Darboux vector $W$ of a Frenet curve, in [8]. Also, they introduced new associated curves in Euclidean 4 -space $\mathbb{E}^{4}$. Furthermore, associated curves of a curve were studied in Minkowski 3-space and three dimensional Lie groups by some authors $[2,3,5,10,11]$.

In this paper, some new associated curves of a given curve by utilizing the alternative frame $\{N, C, W\}$ defined by [13] are introduced and some characterizations related to them are given.

\section{Background on curves in Euclidean 3-space}

In this section, we recall some basic notions and definitions. We are given a spatial regular curve in $\mathbb{E}^{3}$

$$
\alpha=\alpha(s): I \subset \mathbb{R} \rightarrow \mathbb{E}^{3}
$$

where $s$ is the arc-length parameter, that is, $\left\|\alpha^{\prime}(s)\right\|=1$. Because $\alpha(s)$ is a regular curve, the Frenet frame given by $T(s)=\alpha^{\prime}(s), N(s)=\frac{\alpha^{\prime \prime}(s)}{\left\|\alpha^{\prime \prime}(s)\right\|}$ and $B(s)=T(s) \times N(s)$, is well-defined along the curve $\alpha(s)$, where $T(s), N(s)$ 
and $B(s)$ are called the tangent vector, the principal normal vector and the binormal vector of the curve $\alpha$, respectively. Then, the Frenet equations are given by the following relations:

$$
\frac{d}{d s}\left(\begin{array}{l}
T(s) \\
N(s) \\
B(s)
\end{array}\right)=\left(\begin{array}{ccc}
0 & \kappa(s) & 0 \\
-\kappa(s) & 0 & \tau(s) \\
0 & -\tau(s) & 0
\end{array}\right)\left(\begin{array}{c}
T(s) \\
N(s) \\
B(s)
\end{array}\right)
$$

where $\kappa(s)$ and $\tau(s)$ are called the curvature and the torsion of the curve, respectively, and defined by

$$
\kappa(s)=\left\|\alpha^{\prime \prime}(s)\right\|, \quad \tau(s)=\frac{\operatorname{det}\left(\alpha^{\prime}(s), \alpha^{\prime \prime}(s), \alpha^{\prime \prime \prime}(s)\right.}{\left\|\alpha^{\prime \prime}(s)\right\|^{2}} .
$$

A new alternative frame $\{N, C, W\}$ has been introduced by Uzunoglu et al. [13], where $N, C=\frac{N^{\prime}}{\left\|N^{\prime}\right\|}$ and $W=\frac{\tau T+\kappa B}{\sqrt{\tau^{2}+\kappa^{2}}}$ are the unit principal normal, the derivative of the principal normal and the Darboux vector field along the curve, respectively. The derivative formulas of the alternative frame are governed by the relations:

$$
\frac{d}{d s}\left(\begin{array}{c}
N(s) \\
C(s) \\
W(s)
\end{array}\right)=\left(\begin{array}{ccc}
0 & f(s) & 0 \\
-f(s) & 0 & g(s) \\
0 & -g(s) & 0
\end{array}\right)\left(\begin{array}{c}
N(s) \\
C(s) \\
W(s)
\end{array}\right)
$$

where $f(s)=\sqrt{\kappa^{2}+\tau^{2}}$ and $g(s)=\frac{\kappa^{2}\left(\frac{\tau}{\kappa}\right)^{\prime}}{\kappa^{2}+\tau^{2}}$ some differentiable functions.

Definition 2.1 ( $C$-slant helix). Let $\alpha(s)$ be a unit-speed curve with the alternative frame $\{N, C, W\}$. The curve $\alpha(s)$ is called a $C$-slant helix if the vector field $C$ makes a constant angle $\varphi\left(\varphi \neq \frac{\pi}{2}\right)$ with a fixed direction along the curve, [13].

A useful result for $C$-slant helix has been given by Uzunoglu et al. in [13]. They have proved that the curve $\alpha$ is a $C$-slant helix if and only if the following relation is satisfied:

$$
\Gamma=\frac{f^{2}\left(\frac{g}{f}\right)^{\prime}}{\left(f^{2}+g^{2}\right)^{3 / 2}}=\frac{\sigma^{\prime}}{f\left(1+\sigma^{2}\right)^{3 / 2}}=\text { const } .
$$

Definition 2.2 ( $W$-direction curves). Consider a Frenet curve $\alpha$ and the unit Darboux vector $W$ of $\alpha$. A curve $\tilde{\alpha}$ is called a $W$-direction curve, if the curve $\tilde{\alpha}$ is an integral curve of $W,[8]$. 


\section{Associated curves according to the alternative frame $\{N, C, W\}$}

In this section, we introduce new associated curves in terms of the alternative frame and give some related results.

Let $\alpha=\alpha(s)$ be a unit speed curve in $\mathbb{E}^{3}$. The Darboux vector of $\alpha$ in terms of the alternative frame $\{N, C, W\}$ is given by

$$
D=\frac{\sigma N+W}{\sqrt{1+\sigma^{2}}}
$$

which provides $D \times N=N^{\prime}, D \times C=C^{\prime}$ and $D \times W=W^{\prime}$, where $\times$ denotes the vector product in $\mathbb{E}^{3},[13]$.

Definition 3.1. We are given a unit-speed curve $\alpha(s): I \subset \mathbb{R} \rightarrow \mathbb{E}^{3}$ and the Darboux vector field $D$ in terms of the alternative moving frame $\{N, C, W\}$ of the curve. D-direction curve $\bar{\alpha}$ is defined as an integral curve of $D(s)$, that is

$$
\bar{\alpha}(s)=\int D(s) d s .
$$

Theorem 3.1. Let $\alpha(s): I \subset \mathbb{R} \rightarrow \mathbb{E}^{3}$ be a unit-speed curve and $\bar{\alpha}$ be the $D$-direction curve of the curve $\alpha$. Then, the necessary and sufficient condition for the curve $\alpha$ to be a slant helix is the D-direction curve $\bar{\alpha}$ to be a straight line.

Proof. If the curve $\alpha$ is a slant helix, then from Eq. (1.2) $\sigma=$ const. According to the definition of $D$-direction curve, we can write

$$
\bar{\alpha}^{\prime}(s)=D(s)=\frac{1}{\sqrt{1+\sigma^{2}}}(\sigma N+W) .
$$

Differentiating Eq. (3.2), we obtain $\bar{\alpha}^{\prime \prime}(s)=0$ and thus $\alpha(s)=a s+b(a, b \in \mathbb{R}$ constants). This means that $\bar{\alpha}$ is a straight line.

Conversely, let $\bar{\alpha}$ be a straight line, that is $\bar{\alpha}(s)=a s+b$ for some $a, b \in \mathbb{R}$ constants. In this case, it can be easily obtained that $\bar{\alpha}^{\prime}(s)=D(s)=a$ (const.). Differentiating this equality, we get

$$
\bar{\alpha}^{\prime \prime}(s)=D^{\prime}(s)=\frac{\sigma^{\prime}(s)}{\left(1+\sigma^{2}(s)\right)^{3 / 2}} N-\frac{\sigma(s) \sigma^{\prime}(s)}{\left(1+\sigma^{2}(s)\right)^{3 / 2}} W=0 .
$$

Thus, we obtain $\sigma^{\prime}(s)=0$, that is $\sigma(s)=$ const. This completes the proof.

Theorem 3.2. Consider a unit-speed curve $\alpha$ and the D-direction curve $\bar{\alpha}$ of $\alpha$. Providing that $\alpha$ is not a slant helix, the functions $\bar{f}$ and $\bar{g}$ are given as

$$
\bar{g}=-\frac{\Gamma^{\prime}}{1+\Gamma^{2}}, \quad \bar{f}=\frac{\sigma^{\prime}}{\Gamma\left(1+\sigma^{2}\right)} \sqrt{\Gamma^{2}+1} .
$$


Proof. Let $\alpha$ be a unit speed curve and $\bar{\alpha}$ be the $D$-direction curve of $\alpha$. From the definition of $D$-direction curve, we can write $\bar{\alpha}(s)=\int D(s) d s$, that is,

$$
\bar{\alpha}^{\prime}=\bar{T}=D=\frac{\sigma}{\sqrt{1+\sigma^{2}}} N+\frac{1}{\sqrt{1+\sigma^{2}}} W
$$

If we differentiate the last equation, we can easily obtain

$$
\bar{\kappa} \bar{N}=\frac{\sigma^{\prime}}{\left(1+\sigma^{2}\right)^{3 / 2}} N-\frac{\sigma \sigma^{\prime}}{\left(1+\sigma^{2}\right)^{3 / 2}} W .
$$

Taking norm, we immediately get

$$
\bar{\kappa}=\frac{\left|\sigma^{\prime}\right|}{1+\sigma^{2}} .
$$

Then, without loss of generality we can take $\sigma^{\prime}>0$, that is, $\bar{\kappa}=\frac{\sigma^{\prime}}{1+\sigma^{2}}$. Hence, after routine computations we find the principal normal and binormal vector fields of $\bar{\alpha}$ as

$$
\begin{aligned}
\bar{N} & =\frac{1}{\sqrt{1+\sigma^{2}}} N-\frac{\sigma}{\sqrt{1+\sigma^{2}}} W, \\
\bar{B} & =\bar{T} \times \bar{N}=C .
\end{aligned}
$$

which yields

$$
\bar{\tau}=f \sqrt{1+\sigma^{2}} .
$$

On the other hand, we know that $\bar{W}=\frac{\bar{H} \bar{T}+\bar{B}}{\sqrt{1+\bar{H}^{2}}}$. Differentiating this equality, it is easy to obtain that

$$
-\bar{g} \bar{C}=\frac{\bar{H}^{\prime}}{\left(1+\bar{H}^{2}\right)^{3 / 2}} \bar{T}-\frac{\bar{H} \bar{H}^{\prime}}{\left(1+\bar{H}^{2}\right)^{3 / 2}} \bar{B},
$$

By taking norm in the last equation, we have

$$
\bar{g}=\frac{\bar{H}^{\prime}}{1+\bar{H}^{2}} .
$$

From the alternative frame, we have $\bar{f}=\sqrt{\bar{\kappa}^{2}+\bar{\tau}^{2}}$, jointly with Eqs. (3.4) and (3.7) yield

$$
\bar{g}=\frac{\left(\frac{f\left(1+\sigma^{2}\right)^{3 / 2}}{\sigma^{\prime}}\right)^{\prime}}{1+\frac{f^{2}\left(1+\sigma^{2}\right)^{3}}{\left(\sigma^{\prime}\right)^{2}}}, \quad \bar{f}=\frac{1}{1+\sigma^{2}} \sqrt{\left(\sigma^{\prime}\right)^{2}+f^{2}\left(1+\sigma^{2}\right)^{3}} .
$$


Then, using the Eq. (2.3) we obtain Eq. (3.3).

Theorem 3.3. Let $\alpha(s)$ be a unit-speed curve and $\bar{\alpha}$ be the D-direction curve of $\alpha$. The necessary and sufficient condition for the curve $\alpha$ to be a $C$-slant helix is the D-direction curve $\bar{\alpha}$ to be a general helix.

Proof. Let $\alpha$ be a $C$-slant helix in $\mathbb{E}^{3}$. From (2.2), we have

$$
\frac{f\left(1+\sigma^{2}\right)^{3 / 2}}{\sigma^{\prime}}=c \text { (const.). }
$$

Hence, by Eqs. (3.4) and (3.7) one can easily obtain

$$
\frac{f\left(1+\sigma^{2}\right)^{3 / 2}}{\sigma^{\prime}}=\frac{f \sqrt{1+\sigma^{2}}}{\frac{\sigma^{\prime}}{1+\sigma^{2}}}=\frac{\bar{\tau}}{\bar{\kappa}}=c,
$$

then $\bar{\alpha}$ is a general helix.

Conversely, if $\bar{\alpha}$ is a general helix, then we know that $\frac{\bar{\tau}}{\bar{\kappa}}=c$ (const.). Using again Eqs. (3.4) and (3.7), we get

$$
\frac{\bar{\tau}}{\bar{\kappa}}=\frac{f\left(1+\sigma^{2}\right)^{3 / 2}}{\sigma^{\prime}}=c,
$$

that is, the curve $\alpha$ is a $C$-slant helix. This completes the proof.

Corollary 3.1. Let $\alpha$ be a unit-speed curve, $\bar{\alpha}$ be the $D$-direction curve of $\alpha$ and $\widetilde{\bar{\alpha}}$ be the $W$-direction curve of $\bar{\alpha}$. If the curve $\alpha$ is a $C$-slant helix, then the curve $\widetilde{\bar{\alpha}}$ is a straight line.

Proof. The theorem can be easily proved by Corollary 1 in [8] and Theorem 3.3

Definition 3.2. Let $\alpha$ be a unit-speed curve with the alternative frame $\{N, C, W\}$. $A$ curve $\widehat{\alpha}$ is called the $C$-direction curve of the curve $\alpha$ if it is an integral curve of $C$, that is, $\widehat{\alpha}=\int C(s) d s$.

Theorem 3.4. Let us consider a unit-speed curve $\alpha$ with the alternative frame $\{N, C, W\}$ in $\mathbb{E}^{3}$ and the $C$-direction curve $\widehat{\alpha}$ of $\alpha$. The curve $\alpha$ is a $C$-slant helix if and only if the $C$-direction curve $\widehat{\alpha}$ is a helix.

Proof. Let $\alpha$ be a unit-speed curve in $\mathbb{E}^{3}$ and $\widehat{\alpha}$ be the $C$-direction curve of $\alpha$. From Def. 3.2, we have $\widehat{\alpha}^{\prime}(s)=\widehat{T}(s)=C(s)$. Differentiating this equality and using Eqs. (2.1) and (2.2), we easily obtain

$$
\widehat{\alpha}^{\prime \prime}(s)=\widehat{\kappa}(s) \widehat{N}(s)=-f(s) N(s)+g(s) W(s),
$$


which yields $\widehat{\kappa}=\sqrt{f^{2}+g^{2}}=f \sqrt{1+\sigma^{2}}$. Thus, the principal normal vector of $\widehat{\alpha}$ can be easily obtained as

$$
\widehat{N}=\frac{-1}{\sqrt{1+\sigma^{2}}} N+\frac{\sigma}{\sqrt{1+\sigma^{2}}} W .
$$

Then, using cross product in $\mathbb{E}^{3}$, one can easily show that the binormal vector $\widehat{B}$ of $\widehat{\alpha}$ is found as

$$
\widehat{B}=\frac{\sigma}{\sqrt{1+\sigma^{2}}} N+\frac{1}{\sqrt{1+\sigma^{2}}} W .
$$

If we differentiate the last equation, we directly get $\widehat{\tau}=\frac{\sigma^{\prime}}{1+\sigma^{2}}$. Thus, we have the following relation:

$$
\widehat{H}=\frac{\widehat{\tau}}{\widehat{\kappa}}=\frac{\sigma^{\prime}}{f\left(1+\sigma^{2}\right)^{3 / 2}} .
$$

This means that $\alpha$ is a $C$-slant helix if and only if the $C$-direction curve of $\alpha$ is a helix.

Corollary 3.2. Let $\alpha(s)$ be a unit-speed curve in terms of the alternative frame and $\widehat{\alpha}$ be the $C$-direction curve of $\alpha$ in $\mathbb{E}^{3}$. Then, $\alpha$ is a slant helix iff $\widehat{\alpha}$ is a plane curve.

Proof. From the computations in Theorem 3.4, we have

$$
\widehat{H}=\frac{\sigma^{\prime}}{f\left(1+\sigma^{2}\right)^{3 / 2}} .
$$

If $\alpha$ is a slant helix, then $\sigma$ is a constant. Hence, using the last equation we get

$$
\widehat{H}=\frac{\widehat{\tau}}{\widehat{\kappa}}=0
$$

that is, $\widehat{\alpha}$ is a plane curve.

Conversely, if $\widehat{\alpha}$ is a plane curve, then $\widehat{\tau}=0$. Therefore, from Eq. (3.11) we obtain $\sigma^{\prime}=0$, that is $\sigma=c$ (const.). Then, $\alpha$ is a slant helix.

Now, we introduce some new associated curves depending on the alternative frame $\{N, C, W\}$ of a given curve.

Definition 3.3. Let $\alpha$ be a unit-speed curve in terms of the alternative frame apparatus $\{N, C, W, f, g\}$.

(i) If the position vectors of a curve $\beta$ always lie on the plane spanned by the vectors $N$ and $C$, then the curve $\beta$ is called $a(N, C)$ plane curve of $\alpha$. 
(ii) If the position vectors of a curve $\beta$ always lie on the plane spanned by the vectors $N$ and $W$, then the curve $\beta$ is called a $(N, W)$ plane curve of $\alpha$.

(iii) If the position vectors of a curve $\beta$ always lie on the plane spanned by the vectors $C$ and $W$, then the curve $\beta$ is called a $(C, W)$ plane curve of $\alpha$.

Theorem 3.5. Consider a unit-speed curve $\alpha(s)$ with the alternative frame $\{N, C, W\}$ and the $N$-direction curve $\breve{\alpha}(s)$ of $\alpha(s)$ in $\mathbb{E}^{3}$.

(i) If the curve $\breve{\alpha}(s)$ is a $(N, W)$ plane curve of $\alpha$, then $\sigma(s)$ is a linear function.

(ii) If the curve $\breve{\alpha}$ is a $(C, W)$ plane curve of $\alpha$, then the following relationship is satisfied:

$$
a f \cos \left(\int g d s\right)-b f \sin \left(\int g d s\right)=1, \quad a, b \in \mathbb{R}
$$

(iii) If the curve $\breve{\alpha}$ is a $(N, C)$ plane curve of $\alpha$, then $\alpha$ is a general helix or a straight line.

Proof. (i) From Def. 3.3, we can write $\breve{\alpha}(s)=\lambda(s) N(s)+\mu(s) W(s)$, where $\lambda(s)$ and $\mu(s)$ nonzero functions. Differentiating the last equation with respect to $s$ and using the alternative frame equations, we get

$$
\breve{\alpha}^{\prime}=\breve{T}=N=\lambda^{\prime} N+(\lambda f-\mu g) C+\mu^{\prime} W
$$

then,

$$
\begin{aligned}
& \lambda^{\prime}=1 \\
& \lambda f-\mu g=0 \\
& \mu^{\prime}=0
\end{aligned}
$$

The last equations give us $\frac{g}{f}=a s+b$, that is, $\sigma$ is a linear function, where $a$ and $b$ are real constants.

(ii) Let the $N$-direction curve $\breve{\alpha}$ be a $(C, W)$ plane curve of $\alpha$, then from Def. 3.3 , we can write $\breve{\alpha}=\int N d s=\lambda(s) C(s)+\mu(s) W(s)$. Differentiating this equality and using the alternative frame equations $(2.2)$, we get

$$
\breve{T}=N=-\lambda f N+\left(\lambda^{\prime}-\mu g\right) C+\left(\mu^{\prime}+\lambda g\right) W
$$


then

$$
-\lambda f=1, \quad \lambda^{\prime}-\mu g=0, \quad \mu^{\prime}+\lambda g=0 .
$$

Using the first and second equation in (3.12), we directly obtain

$$
\lambda=\frac{-1}{f} \quad \mu=-\frac{1}{g}\left(\frac{1}{f}\right)^{\prime},
$$

that is,

$$
\breve{\alpha}=\frac{-1}{f} C-\frac{1}{g}\left(\frac{1}{f}\right)^{\prime} W .
$$

Moreover, from the first and third equation in (3.12), we can write

$$
\left[\frac{-1}{g}\left(\frac{1}{f}\right)^{\prime}\right]^{\prime}=\frac{g}{f}
$$

Taking $\frac{1}{f}=h(s)$ and $-\frac{1}{g}=r(s)$, Eq. (3.13) can be reconstructed as

$$
\left[r(s) h^{\prime}(s)\right]^{\prime}+\frac{h(s)}{r(s)}=0 .
$$

For solving this differential equation if we apply a change of variable $t=\int \frac{1}{r(s)} d s$, we obtain

$$
\frac{d^{2} h}{d t^{2}}+h=0
$$

Then, solving the last differential equation with constant coefficients, we find

$$
h(t)=a \cos t+b \sin t, \quad a, b \in \mathbb{R},
$$

which yields

$$
a f \cos \left(\int g d s\right)-b f \sin \left(\int g d s\right)=1 .
$$

which completes the proof.

(iii) Let the $N$-direction curve $\widehat{\alpha}(s)$ be a $(N, C)$ plane curve of $\alpha$, then we can write $\widehat{\alpha}(s)=\int N(s) d s=\lambda(s) N(s)+\mu(s) C(s)$. Differentiating this equality and using the alternative frame equations, we get

$$
\widehat{T}=N=\left(\lambda^{\prime}-\mu f\right) N+\left(\lambda f+\mu^{\prime}\right) C+g \mu W,
$$

then

$$
\lambda^{\prime}-\mu f=1, \quad \lambda f+\mu^{\prime}=0, \quad g \mu=0 .
$$


In case of $\mu=0$, we obtain $f=0$, then we get $\kappa=\tau=0$. This means that the curve $\nu$ is a straight line.

In case of $g=0$, the curve $\nu$ is a general helix

This completes the proof.

Theorem 3.6. Let $\alpha$ be a unit-speed curve with the alternative frame $\{N, C, W\}$ and $\widehat{\alpha}$ be the $C$-direction curve of $\alpha$ in $\mathbb{E}^{3}$.

(i) If the curve $\widehat{\alpha}$ is a $(N, W)$ plane curve of $\alpha$, then the following relation is satisfied:

$$
c_{1} f(s)+c_{2} g(s)=1, \quad c_{1}, c_{2} \in \mathbb{R} \text {. }
$$

(ii) If the curve $\widehat{\alpha}$ is a $(C, W)$ plane curve, then the function $g$ is a constant or $\alpha$ is a straight line.

(iii) If the curve $\widehat{\alpha}$ is a $(N, C)$ plane curve of $\alpha$, then $\alpha$ is a general helix or the function $f$ is a constant.

Proof. (i) It can be proved by the method used in Theorem 3.5 (i).

(ii) Let the $C$-direction curve $\widehat{\alpha}$ be a $(C, W)$ plane curve, then from Def. 3.3, we can write $\widehat{\alpha}=\int C d s=\lambda(s) C(s)+\mu(s) W(s)$. Differentiating this equality and using the alternative frame equations (2.2), we get

$$
\widehat{T}=C=-\lambda f N+\left(\lambda^{\prime}-\mu g\right) C+\left(\mu^{\prime}+\lambda g\right) W,
$$

then

$$
-\lambda f=0, \quad \lambda^{\prime}-\mu g=1, \quad \mu^{\prime}+\lambda g=0 .
$$

Hence, we have three cases:

(a) If $\lambda=0$ and $f \neq 0$, then from the third equation in (3.14) we obtain $\mu=c$ (const.). Substituting $\lambda=0$ and $\mu=c$ into the second equation in (3.14), we find $g=-\frac{1}{c}$ (const.).

(b) If $\lambda \neq 0$ and $f=0$, then we get $\kappa=\tau=0$. This means that the curve $\alpha$ is a straight line.

(c) If $\lambda=f=0$, then the curvature $g$ is equal to zero. However, from the second equation in (3.14) we know $-\mu g=1$. Hence, this is a contradiction.

(iii) It can be proved similar to Theorem 3.5 (iii). 
Theorem 3.7. Let $\alpha$ be a unit-speed curve and $\widetilde{\alpha}$ be the $W$-direction curve of $\alpha$ with the alternative frame $\{N, C, W\}$ in $\mathbb{E}^{3}$.

(i) If the curve $\widetilde{\alpha}$ is a $(N, W)$ plane curve of $\alpha$, then $\sigma(s)=\frac{1}{a s+b}$, where $a, b \in \mathbb{R}$.

(ii) If the curve $\widetilde{\alpha}$ is a $(C, W)$ plane curve of $\alpha$, then $\alpha$ is a general helix or a straight line.

(iii) If the curve $\widetilde{\alpha}$ is a $(N, C)$ plane curve of $\alpha$, then the functions $f$ and $g$ satisfy the following relation:

$$
a g \cos \left(\int f d s\right)-b g \sin \left(\int f d s\right)=1, \quad a, b \in \mathbb{R}
$$

Proof. It can be proved by the method used in Theorem 3.5.

Theorem 3.8. We are given a unit- speed curve $\alpha$ with the alternative frame $\{N, C, W\}$ and $\bar{\alpha}$ be the $D$-direction curve of $\alpha$.

(i) If $\bar{\alpha}$ is a $(N, W)$ plane curve of $\alpha$, then the curve $\alpha$ is a slant helix.

(ii) If $\bar{\alpha}$ is a $(C, W)$ plane curve of $\alpha$, then the following differential equation is satisfied:

$$
p^{\prime}=g \int \sqrt{1+\sigma^{2}} d s
$$

where $p=-\frac{\sigma}{f \sqrt{1+\sigma^{2}}}$.

(iii) If $\bar{\alpha}$ is a $(N, C)$ plane curve of $\alpha$, then the functions $f$ and $g$ satisfy the following differential equation:

$$
q^{\prime}=-f \int \frac{\sqrt{1+\sigma^{2}}}{\sigma} d s
$$

where $q=\frac{1}{g \sqrt{1+\sigma^{2}}}$.

Proof. (i) From Def. 3.3, we have

$$
\bar{\alpha}=\lambda(s) N(s)+\mu(s) W(s),
$$

where $\{N, C, W\}$ is the alternative frame along the curve $\alpha, \lambda(s)$ and $\mu(s)$ are some nonzero functions. If we differentiate the last equation, we directly obtain

$$
\bar{T}=\lambda^{\prime} N+(\lambda f-\mu g) C+\mu^{\prime} W .
$$


Moreover, from Def. 3.1 we know that $D=\bar{\alpha}^{\prime}=\bar{T}$. Hence, substituting $\bar{T}=D=\frac{\sigma N+W}{\sqrt{1+\sigma^{2}}}$ into Eq. (3.16), we get

$$
\frac{\sigma}{\sqrt{1+\sigma^{2}}} N+\frac{1}{\sqrt{1+\sigma^{2}}} W=\lambda^{\prime} N+(\lambda f-\mu g) C+\mu^{\prime} W,
$$

then,

$$
\lambda^{\prime}=\frac{\sigma}{\sqrt{1+\sigma^{2}}}, \quad \mu^{\prime}=\frac{1}{\sqrt{1+\sigma^{2}}}, \quad \lambda f-\mu g=0 .
$$

Utilizing these three equations, it is easy to obtain that $\frac{\lambda^{\prime}}{\mu^{\prime}}=\sigma=\frac{\lambda}{\mu}=$ $c$ (const.), that is, $\alpha$ is a slant helix.

(ii) Assume that the $D$-direction curve $\bar{\alpha}$ is the $(C, W)$ plane curve. Utilizing Def. 3.3, we have $\bar{\alpha}=\int D d s=\lambda C+\mu W$. Differentiating this equality we get

$$
\bar{T}=D=\frac{\sigma N+W}{\sqrt{1+\sigma^{2}}}=-\lambda f N+\left(\lambda^{\prime}-\mu g\right) C+\left(\mu^{\prime}+\lambda g\right) W,
$$

then,

$$
-\lambda f=\frac{\sigma}{\sqrt{1+\sigma^{2}}}, \quad \lambda^{\prime}-\mu g=0, \quad \mu^{\prime}+\lambda g=\frac{1}{\sqrt{1+\sigma^{2}}} .
$$

From the first equation in (3.18), we obtain $\lambda=-\frac{\sigma}{f \sqrt{1+\sigma^{2}}}$. Substituting this equality into the third equation in (3.18), one can easily obtain $\mu^{\prime}=\sqrt{1+\sigma^{2}}$, that is $\mu=\int \sqrt{1+\sigma^{2}} d s$. Thus, using the second equation in $(3.18)$ we find

$$
\left(-\frac{\sigma}{f \sqrt{1+\sigma^{2}}}\right)^{\prime}=g \int \sqrt{1+\sigma^{2}} d s
$$

which concludes the proof.

(iii) It can be proved similar to (ii).

\section{Examples}

Example 4.1. Consider a unit-speed slant helix

$$
\alpha(s)=\left(-\frac{1}{12} \cos 4 s-\frac{1}{3} \cos 2 s,-\frac{1}{12} \sin 4 s-\frac{1}{3} \sin 2 s,-\frac{2 \sqrt{2}}{3} \cos s\right) .
$$




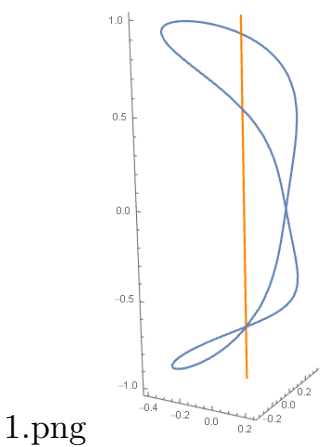

Figure 1: The slant helix $\alpha$ (Red) and its $D$-direction curve $\bar{\alpha}$ (Blue) for $\lambda_{1}=\lambda_{2}=\lambda_{3}=0$.

After simple computation, the alternative frame apparatus of the curve $\alpha$ is obtained as follows:

$$
\left\{\begin{array}{l}
N(s)=\left(\frac{2 \sqrt{2}}{3} \cos 3 s, \frac{2 \sqrt{2}}{3} \sin 3 s, \frac{1}{3}\right), \\
C(s)=(-\sin 3 s, \cos 3 s, 0), \\
W(s)=\left(-\frac{1}{3} \cos 3 s,-\frac{1}{3} \sin 3 s, \frac{2 \sqrt{2}}{3}\right), \\
f(s)=2 \sqrt{2}, g(s)=1 .
\end{array}\right.
$$

Moreover, the Darboux vector in terms of the alternative frame $\{N, C, W\}$ is calculated as

$$
D(s)=(0,0,1) .
$$

Thus, the D-direction curve of the slant helix $\alpha$ is obtained as follows:

$$
\bar{\alpha}=\left(\lambda_{1}, \lambda_{2}, s+\lambda_{3}\right) ; \quad \lambda_{1}, \lambda_{2}, \lambda_{3} \in \mathbb{R}
$$

which satisfies Theorem 3.1, (Figure 1)

Example 4.2. In this example, firstly we construct a $C$-slant helix with an approximation using Taylor expansion. The fundamental theorem of curves states that there exists a unique regular parametrized curve $\alpha$ that has specific curvature and torsion with a solution of differential equation. D. J. Struik [12] has given the following form of a curve using the Frenet vectors of the curve 
and Taylor expansion:

$$
\alpha(s)=\alpha(0)+\left(s-\frac{s^{3} \kappa^{2}}{6}\right) T+\left(\frac{s^{2} \kappa}{2}-\frac{s^{3} \kappa^{\prime}}{6}\right) N+s-\frac{s^{3} \kappa \tau}{6} B+o\left(s^{3}\right)
$$

Uzunoglu et al. [13] obtained the curvature $\kappa(s)$ and the torsion $\tau(s)$ of a $C$-slant helix. Then, taking $\kappa(s)=\frac{1}{\sqrt{2}} \cos \left(\frac{s}{\sqrt{2}}\right) \cos \left(\cos \frac{s}{\sqrt{2}}\right)$,

$\tau(s)=-\frac{1}{\sqrt{2}} \cos \left(\frac{s}{\sqrt{2}}\right) \sin \left(\cos \frac{s}{\sqrt{2}}\right)$ and using the last equation, we get the $C$-slant helix $\alpha(s)$ given in Figure 2 (Blue). After some computations, the alternative frame apparatus of $\alpha$ is obtained as

$$
\left\{\begin{aligned}
N(s)= & \left(\cos \frac{s}{\sqrt{2}} \cos s+\frac{1}{\sqrt{2}} \sin \frac{s}{\sqrt{2}} \sin s, \cos \frac{s}{\sqrt{2}} \sin s-\frac{1}{\sqrt{2}} \sin \frac{s}{\sqrt{2}} \cos s\right. \\
& \left.\frac{1}{\sqrt{2}} \sin \frac{s}{\sqrt{2}}\right) \\
C(s)= & \left(-\frac{1}{\sqrt{2}} \sin s, \frac{1}{\sqrt{2}} \cos s, \frac{1}{\sqrt{2}}\right) \\
W(s)= & \left(-\sin \frac{s}{\sqrt{2}} \cos s+\frac{1}{\sqrt{2}} \cos \frac{s}{\sqrt{2}} \sin s,\right. \\
& \left.-\sin \frac{s}{\sqrt{2}} \sin s-\frac{1}{\sqrt{2}} \cos \frac{s}{\sqrt{2}} \cos s, \frac{1}{\sqrt{2}} \cos \frac{s}{\sqrt{2}}\right) \\
f(s)= & \frac{1}{\sqrt{2}} \cos \frac{s}{\sqrt{2}}, g(s)=\frac{1}{\sqrt{2}} \sin \frac{s}{\sqrt{2}} .
\end{aligned}\right.
$$

Thus, the $C$-direction curve of the $C$-slant helix $\alpha$ is obtained as

$$
\bar{\alpha}=\left(\frac{1}{\sqrt{2}} \cos s+\lambda_{1}, \frac{1}{\sqrt{2}} \sin s+\lambda_{2}, \frac{s}{\sqrt{2}}+\lambda_{3}\right),
$$

which satisfies Theorem 3.4. 


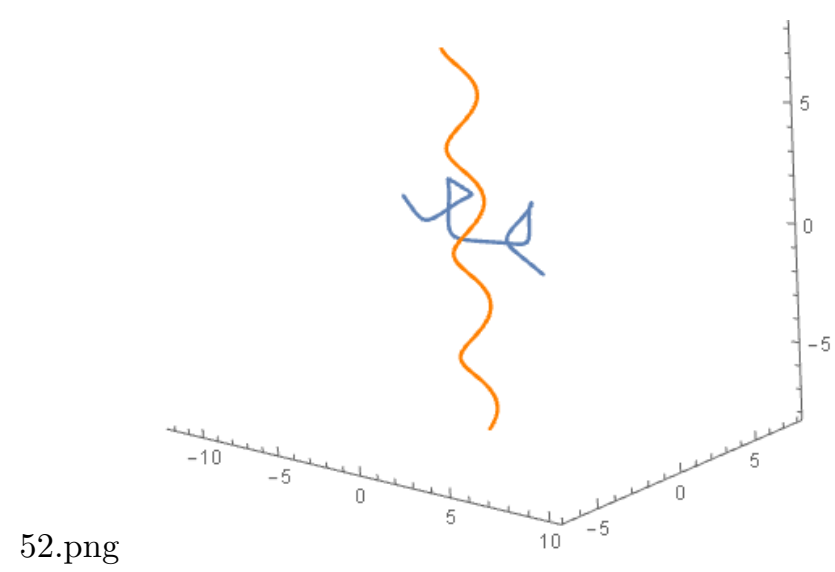

Figure 2: The $C$-slant helix $\alpha$ (Blue) and its $D$-direction curve $\bar{\alpha}$ as a general helix (Orange) for $\lambda_{1}=\lambda_{2}=\lambda_{3}=0$.

Example 4.3. Let $\alpha(s)=\left(a_{1}(s), a_{2}(s), a_{3}(s)\right)$ be a unit-speed curve of constant precession [14], where

$$
\begin{aligned}
& a_{1}(s)=\frac{(\sqrt{2}+1)^{2}}{2 \sqrt{2}} \sin [(\sqrt{2}-1) s]-\frac{(\sqrt{2}-1)^{2}}{2 \sqrt{2}} \sin [(\sqrt{2}+1) s] \\
& a_{2}(s)=-\frac{(\sqrt{2}+1)^{2}}{2 \sqrt{2}} \cos [(\sqrt{2}-1) s]+\frac{(\sqrt{2}-1)^{2}}{2 \sqrt{2}} \cos [(\sqrt{2}+1) s] \\
& a_{3}(s)=\frac{1}{\sqrt{2}} \sin s .
\end{aligned}
$$

Then, it is easy to show that the alternative frame apparatus of the curve $\alpha$ is computed as follows:

$$
\left\{\begin{array}{l}
N(s)=\left(\frac{1}{\sqrt{2}} \cos \sqrt{2} s, \frac{1}{\sqrt{2}} \sin \sqrt{2} s,-\frac{1}{\sqrt{2}}\right), \\
C(s)=(-\sin \sqrt{2} s, \cos \sqrt{2} s, 0), \\
W(s)=\left(\frac{1}{\sqrt{2}} \cos \sqrt{2} s, \frac{1}{\sqrt{2}} \sin \sqrt{2} s, \frac{1}{\sqrt{2}}\right), \\
f(s)=1, g(s)=-1 .
\end{array}\right.
$$

If we choose $\lambda=2, \mu=-1$, then the $C$-direction curve $\widehat{\alpha}$ as $(N, W)$ plane 
curve is found as

$$
\widehat{\alpha}(s)=\left(\frac{1}{\sqrt{2}} \cos \sqrt{2} s+\lambda_{1}, \frac{1}{\sqrt{2}} \sin \sqrt{2} s+\lambda_{2},-\frac{3}{\sqrt{2}}+\lambda_{3}\right)
$$

which satisfies Theorem 3.6, (Figure 3).

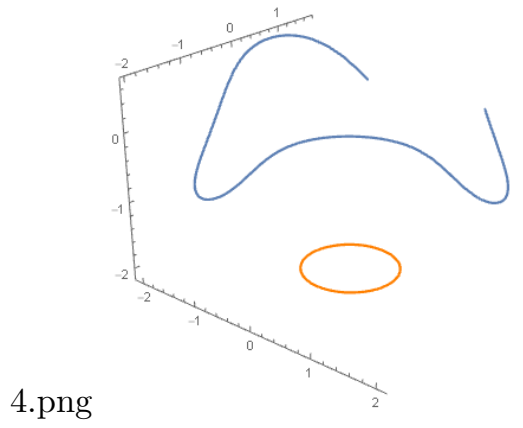

Figure 3: The curve of constant precession $\alpha$ (Blue) and its $C$-direction curve $\widehat{\alpha}$ as a $(N, W)$ plane curve of $\alpha$ (Orange) for $\lambda_{1}=\lambda_{2}=\lambda_{3}=0$.

\section{References}

[1] Choi J and Kim Y, Associated curves of a frenet curve and their applications. Appl. Math. Comput., 2018, 9116-9124 (2012).

[2] Choi J., and Kim Y.H.: Some associated curves of Frenet non-lightlike curves in $\mathbb{E}_{1}^{3}$. J. Math. Anal. Appl. 394, 712-723 (2012).

[3] Gok I, Okuyucu O Z, Yayli Y and Ekmekci N, On Mannheim partner curves in three dimensional Lie groups. Miskolc Math. Notes, 15(2), 467479. (2014).

[4] Izumiya S and Takeuchi N, New special curves and developable surfaces, Turk. J. Math., 28, 153-163 (2004).

[5] Kiziltug S. and Onder M.: Associated curves of Frenet curves in three dimensional compact Lie group, Miskolc Math. Notes, 16, 953-964 (2015).

[6] Kula L and Yayli Y, On Slant Helix and Its Spherical Indicatrix. Appl. Math. Comput., 169, 600-607 (2005).

[7] Lancret M A, Mémoire sur les courbes à double courbure, Mémoires présentés à l'Institut 1, 416-454 (1806). 
[8] Macit N and Duldul M, Some new associated curves of a Frenet curve in $\mathbb{E}^{3}$ and $\mathbb{E}^{4}$, Turk. J. Math., 38, 1023-1037 (2014).

[9] Okuyucu O Z, Gok I, Yayli Y and Ekmekci F N, Slant helices in three dimensional Lie groups. Appl. Math. Comput., 221, 672-683 (2013).

[10] Okuyucu O Z, Gok I, Yayli Y and Ekmekci N, Bertrand curves in three dimensional Lie groups. Miskolc Math. Notes, 17(2), 999-1010 (2016).

[11] Qian J. and Kim Y.H.: Directional associated curves of a null curve in Minkowski 3-space, Bull. Korean Math. Soc., 52, 183-200 (2015).

[12] Struik D., Lectures on Classical Differential Geometry, 2nd ed. Dover: Addison Wesley, 1950.

[13] Uzunoglu B, Gok I and Yayli Y, A new approach on curves of constant precession. Appl. Math. Comput., 275, 317-323 (2016).

[14] Scofield P D, Curves of Constant Precession, Amer. Math. Mon., 102(6), 531-537 (1995).

[15] Ozdamar E and Hacisalihoglu H H, A characterization of inclined curves in Euclidean n-space, Communications de la Faculté des Sciences de l'Université d'Ankara, 24, 15-22 (1975).

Sibel Özdoğan,

Department of Mathematics,

Ankara University,

06100 Ankara, Turkey.

Email: sibellozdogan@gmail.com

O. Oğulcan Tuncer,

Department of Mathematics,

Hacettepe University,

06800 Ankara, Turkey.

Email: otuncer@hacettepe.edu.tr

İsmail Gök,

Department of Mathematics,

Ankara University,

06100 Ankara, Turkey.

Email: igok@science.ankara.edu.tr

Yusuf Yaylı,

Department of Mathematics,

Ankara University,

06100 Ankara, Turkey.

Email: yayli@science.ankara.edu.tr 